\title{
Coronary Graft Anastomoses
}

National Cancer Institute

\section{Source}

National Cancer Institute. Coronary Graft Anastomoses. NCI Thesaurus. Code C80476.

The connection where the graft joins the coronary artery, usually less than or equal to 3

$\mathrm{mm}$ from insertion point. 